Original Research Article

\title{
Effect of anti-epileptic drugs on cognitive functions: a prospective study in individuals with newly diagnosed complex partial seizure and generalized tonic clonic seizure
}

\author{
Jayant Rai*, Preeti P. Yadav, Richa Verma, Mayur Chaudhari
}

Department of Pharmacology, Government Medical College, Surat, Gujarat, India

Received: 17 January 2017 Accepted: 25 February 2017

*Correspondence to:

Dr. Jayant Rai,

Email: raijayant02@gmail.com

Copyright: (C) the author(s), publisher and licensee Medip Academy. This is an openaccess article distributed under the terms of the Creative Commons Attribution NonCommercial License, which permits unrestricted noncommercial use, distribution, and reproduction in any medium, provided the original work is properly cited.

\begin{abstract}
Background: Epilepsy, the third most common neurologic disorder, deteriorates cognitive functions of the patients. Approximately $1 \%$ of the world's population is suffering from epilepsy. Opinions regarding impact of anti-epileptic drugs on cognition are divided. So, this study was designed to assess the impact of anti-epileptic drugs on cognitive performance of patients with complex partial seizure and generalized tonic clonic seizure in Department of Medicine, at Government Medical College, Surat, Gujarat, India.

Methods: In present study, cognitive functions were assessed in 50 patients of newly diagnosed complex partial seizure and generalized tonic clonic seizure coming to the Department of Medicine, Surat. The cognitive functions were evaluated by Addenbrooke's Cognitive Examination (ACE)-III, which assessed memory, attention, fluency, language and visuo-spatial abilities. Follow-up was done after six months of baseline.

Results: Baseline and Follow-up data from 50 patients were analysed. Patient treated with anti-epileptic drugs showed significant improvement in memory, attention, language and visuo-spatial abilities whereas improvement in fluency was not significant. Paired t-test and Wilcoxon Signed Ranks Test were used to analyse the data. For statistical analysis of data SPSS 19.0 software was used.

Conclusions: The available data indicate that the anti-epileptic drugs on shortterm administration do not adversely affect cognitive function in patients with newly diagnosed CPS and GTCS. Importantly, the data suggest that the effects exerted by AEDs could depend on factors linked to patient characteristics and individual susceptibility and to comment on those factors further studies are needed.
\end{abstract}

Keywords: Addenbrooke's cognitive examination-III, Anti-epileptic drugs, CPS, Cognition, Epilepsy, GTCS

\section{INTRODUCTION}

Epilepsy is a chronic condition of recurrent seizures that can also vary from brief and nearly undetectable symptoms to periods of vigorous shaking and convulsions. ${ }^{1}$ Seizures are episodic high-frequency discharge of impulses by a group of neurons in a particular area in the brain. ${ }^{2}$ The electrical balance underlying normal neuronal activity is pathologically altered during seizures-excitation predominates over inhibition. The therapeutic goal of anti-epileptic drugs is to maximize seizure control while minimizing adverse drug effects, thus improving the patient's quality of life. Cognitive dysfunction is seen in a sizeable number of patients with epilepsy. Anti-epileptic drugs are able to improve cognition and behaviour by reducing seizure activity and modifying effects of neurotransmitter.

Several relationships exist between cognitive impairment and epilepsy-related or treatment-related factors. The central cognitive side effect of the anti-epileptic drugs is one of the most important treatment-related factors. ${ }^{3}$ Present study was carried out in the Department of Medicine, Government Medical College, Surat with an 
aim to demonstrate effects of anti-epileptic drugs on cognitive outcomes in newly diagnosed patients with complex partial seizure and generalized tonic-clonic seizure. Objectives in the present study are to analyse the effects of anti-epileptic drugs on cognitive functions of patients with complex partial seizure and generalized tonic clonic seizure in medicine department of New Civil Hospital, Surat, Gujarat, India and to assess five cognitive domains: Attention, memory, verbal fluency, language and visuo-spatial abilities by using Addenbrooke's cognitive examination-III (ACE-III).

\section{METHODS}

This was a single centre prospective, non-interventional and observational study.

\section{Patient selection}

Patients newly diagnosed with complex partial seizure and generalized tonic clonic seizure were enrolled from medicine outpatient department and eligible patients (as per inclusion and exclusion criteria) were included in the study. Patients with newly diagnosed complex partial seizure and generalized tonic clonic seizure were selected as their admission/visiting rate in the medicine department was more as compared to other types of newly diagnosed seizure patients.

\section{Inclusion-exclusion criteria}

Patients of both genders and 13-70 years' age group, newly diagnosed with Complex Partial Seizure and Generalized Tonic Clonic Seizure were included in this study. Patients with Progressive neurological disorder, having history of head injury, alcohol intake, drug abuse, suffering from mental retardation, taking any centrally acting medications, suffering from severe psychiatric problem were excluded from the study.

\section{Study procedure}

The diagnosis of complex partial seizure and generalized tonic clonic seizure was made by the treating Physician of the Department of Medicine, New Civil Hospital, Surat, India.

Data were collected from interviewing the patient as well as from patient's case file maintained in the department of Medicine. The data was recorded in a pre-designed and pre-approved patient data sheet after taking informed consent in written form. The demographic details of the patient and details of the treatment given were duly recorded. The subjects were given participant information number which mentioned confidential details of the subject. Cognitive functions of the recruited patients were evaluated by Addenbrooke's Cognitive Examination-III (ACE-III) for attention, memory, verbal fluency, language and visuo-spatial abilities after fully explaining the testing procedure and getting the patient's consent.
Addenbrooke's Cognition Examination-III was applied to patients for two times, baseline scores were taken after diagnosis was made before starting anti-epileptic drugs and follow-up scores were taken after six months of antiepileptic therapy.

\section{Data analysis}

All values are expressed in terms of mean \pm standard error of mean. For analysis of data distribution Shapiro-Wilk test was done which is a test of normality. Sig (p value) of Shapiro-Wilk test should be $\geq 0.05$ for it to be normally distributed. Comparison of parameters at pre-treatment level and after six months of anti-epileptic drug treatment is done using Paired t-Test and Wilcoxon Signed Ranks Test where $\mathrm{P}$ value $<0.05$ is taken as significant. All statistical analysis is done using SPSS 19.0 software. To assess the data distribution test of normality was done. This test showed that data is normally distributed for memory, and total score. However, data for attention, fluency, visuo-spatial and language score didn't show normal distribution.

\section{RESULTS}

A total of 100 patients diagnosed with complex partial seizure and generalized tonic clonic seizure by the treating physician in Department of Medicine were interviewed and 50 patients were included in study in accordance with inclusion and exclusion criteria. The interview sample comprised of $70 \%(n=35)$ males and $30 \%(n=15)$ females of age ranging from 13 to 70 years and mean age being 30.66 years.

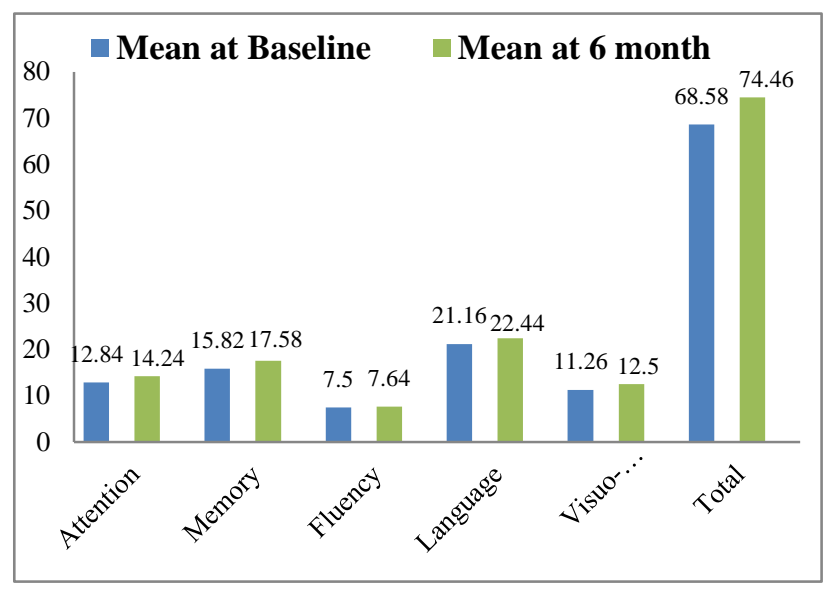

Figure 1: ACE-III score for all variables and total score.

\section{Attention}

Wilcoxon Signed Ranks Test for attention score-revealed that the score at 6 month after anti-epileptic drug treatment had increased values from pre-treatment level values of $12.84 \pm 0.367$ to $14.24 \pm 0.261$ (p-value $<0.05$ ). 


\section{Memory}

On applying Shapiro-Wilk Test, data for memory score showed normal distribution so for the analysis of memory score t-test, which revealed that the score at 6 month after anti-epileptic drug treatment had increased values from pretreatment level values of $15.82 \pm 0.636$ to $17.58 \pm 0.497$ (p-value <0.05).

\section{Fluency}

Wilcoxon Signed Ranks Test for fluency score-revealed that the score at 6 month after anti-epileptic drug treatment was increased values $7.64 \pm 0.314$ (p-value $<0.05)$ as compared to the pre-treatment level values of $7.50 \pm 0.358$

\section{Language}

Wilcoxon Signed Ranks Test for language score-revealed that the score at 6 month after anti-epileptic drug treatment had increased values 22.44 \pm 0.331 (p-value $<0.05)$ as compared to the pre-treatment level values of $21.16 \pm 0.408$.

\section{Visuo-spatial}

Wilcoxon Signed Ranks Test for visuo-spatial scorerevealed that the score at 6 month after anti-epileptic drug treatment had increased values $12.50 \pm 0.348$ (p-value $<0.05)$ as compared to the pre-treatment level values of $11.26 \pm 0.432$.

\section{Total score}

On applying Shapiro-Wilk Test for total score-revealed that the score at 6 month after anti-epileptic drug treatment had increased values from the pre-treatment level values of $68.58 \pm 1.725$ to $74.46 \pm 1.212$ (p-value $<0.05)$.

\section{DISCUSSION}

Epilepsy was documented as a neurological disorder, but there are accumulating evidences that it is a behaviouralcognitive disorder as well. ${ }^{4}$ Anti-epileptic drugs have the potential to impact cognitive functions. Even their modest effects can be of clinical significance and affects patient's life. Epilepsy attracts considerable social stigmatisation all over the world. It is a severe and unpleasant neurologic disorder. It is possible that stigmatisation is greater in developing countries, and is likely to lead to considerable social and psychological morbidity. ${ }^{5}$

Anti-epileptic drugs have the potential to impact cognitive functions..$^{6}$ Although individual sensitivity may lead to severe cognitive effects in individual cases, most studies have not found clinically relevant effects of standard anti-epileptic drugs therapy in study populations. ${ }^{7}$ To control the confounding effects of seizures on cognitive function during baseline, only those patients with two or fewer complex partial seizures and generalized tonic clonic seizures occurring within the three months preceding the study were included.

Neuropsychological testing has been the major method of objectively measuring cognitive function related to the use of anti-epileptic drugs. ${ }^{8}$ The cognitive outcome assessment in this study was done by Addenbrooke's Cognitive Examination-III. All Anti-epileptic drugs employed in the study were well tolerated by patients and the safety profile of anti-epileptic drugs were similar to that reported previously. ${ }^{9}$

Anti-epileptic drugs are given as long-term treatment, and concern about their impact on cognitive function in daily life is an important aspect in the people suffering with epilepsy. ${ }^{8}$ This study didn't assess effectiveness of antiepileptic drugs in people with intellectual disabilities. Recent studies have established that even single seizures can lead to a temporary change in cognitive performance but that changes persist over time only when the frequency of seizures are high or postictal effects are prolonged. $^{10}$

In our study five cognitive domains of every individual enrolled for study were analysed. After six months of treatment with anti-epileptic drugs no significant negative impact on attention was seen. Out of total 50 individuals, 27 out of 35 males and 13 out of 15 females show increase in cognitive score whereas no impact was seen on 6 males and decline was noted in 2 males and 2 females. In our study, after six months of treatment with anti-epileptic drugs increase in cognitive score for memory which includes short-term as well as long-term memory, was seen.

After six months of treatment with anti-epileptic drugs no significant improvement or decline in the cognitive score for fluency was reported. In our study, Anti-epileptic drugs caused improvement of cognitive score for language as tested by cognitive test. In our study, after six months of treatment with anti-epileptic drugs cognitive score for visuo-spatial cognitive domain has shown significant increase.

Cognitive effects of anti-epileptic drugs are among the factors important to consider while deciding therapeutic strategy for management of epilepsy. Seizure type is an important risk factor, which impacts cognitive functions in individuals with complex partial seizures and generalized tonic-clonic seizures. ${ }^{11}$

The potential for anti-epileptic drugs to impact cognition is of significant concern as they are the major therapeutic modality for control of seizures. The anti-epileptic drugs reduce seizures either by enhancing the threshold for noxious stimuli to cause precipitation of seizures or by reducing the excitability of the neurons which can lead to reduction of responses by brain cells which will affect the 
various cognitive functions also. Even their modest effects can be of clinical significant and affect the patient's quality of life. The results of this study provide a support to the fact that anti-epileptic drugs on shortterm therapy don't have any negative impact on cognition.

\section{Limitations}

The strength of the study is that it recruited new-onset cases making it an incidence sample rather than a prevalence sample. Limitation of this study was follow-up of only 6 months, and its unforeseen effects on cognitive function in long term. As anti-epileptic therapy is generally life long, the long-term effects of these drugs on cognition need to be further evaluated. Thus, to generalize the results of this study, study parameters need to be evaluated in larger population and for longer duration.

Funding: No funding sources

Conflict of interest: None declared

Ethical approval: The study was carried out after obtaining the Institutional Ethics Committee clearance letter no MCS/STU/ETHICS/Approval/7879/15 on dated 15/04/2015 and MCS/STU/ETHICS/Approval/23796/15 on dated 17/11/2015 of New Civil Hospital and Government Medical College, Surat, Gujarat

\section{REFERENCES}

1. Guyton AC, Hall JE. The Nervous System: motor and integrative neurophysiology. 16th ed: Elsevier; 2016.

2. Rang HP, Ritter JM, Flower RJ, Henderson G. In Rang \& Dale's Pharmacology. Antiepileptic Drugs. 7 ed: Elsevier; 2012.

3. Lowenstein DH. In Harrison's Principles of Internal Medicine. Seizures and epilepsy. 19th ed. McGraw Hill; 2015.

4. Hanai T. Quality of life in children with epilepsy. Epilepsia. 1996;37:28-32
5. Trimble MR, Krishnamoorthy ES. Neuropsychiatric disorders in epilepsy: some transcultural issues. Epilepsia. 2003;44:21-4.

6. Trimble MR. Anti-epileptic drugs, cognitive function and behavior in children: evidence from recent studies. Epilepsia. 1990;31:S30-4.

7. Donati F, Gobbi G, Campistol J, Rapatz G, Daehler M, Sturm Y et al. The cognitive effects of oxcarbazepine versus carbamazepine or valproate in newly diagnosed children with partial seizures. Seizure. 2007;16(8):670-9.

8. Hessen E, Lossius MI, Reinvang I, Gjerstad L. Influence of major antiepileptic drugs on attention, reaction time, and speed of information processing: results from a randomized, double-blind, placebocontrolled withdrawal study of seizure-free epilepsy patients receiving monotherapy. Epilepsia. 2006;47(12):2038-45.

9. Dam M, Ekberg R, Loyning Y, Waltimo O, Jakobsen $\mathrm{K}$. A double-blind study comparing oxcarbazepine and carbamazepine in patients with newly diagnosed, previously untreated epilepsy. Epilepsy Res. 1989;3(1):70-6.

10. Tromp SC, Weber JW, Aldenkamp AP, Arends J, vander Linden I, Diepman L. Relative influence of epileptic seizures and of epilepsy syndrome on cognitive function. J Child Neurol. 2003;18(6):40712.

11. Aldenkamp AP. Effect of seizures and epileptiform discharges on cognitive function. Epilepsia. 1997;38:s52-s5.

Cite this article as: Rai J, Yadav PP, Verma R, Chaudhari M. Effect of anti-epileptic drugs on cognitive functions: a prospective study in individuals with newly diagnosed complex partial seizure and generalized tonic clonic seizure. Int $\mathrm{J}$ Basic Clin Pharmacol 2017;6:855-8. 